\title{
Boundary regularity result for quasilinear elliptic systems
}

Shuhong Chen ${ }^{1 *}$ and Zhong $\operatorname{Tan}^{2}$

* Correspondence: shiny0320@163. com

${ }^{1}$ Department of Mathematics and Information Science, Zhangzhou Normal University, Zhangzhou 363000, Fujian, China Full list of author information is available at the end of the article

\section{Abstract}

We consider boundary regularity for weak solutions of second-order quasilinear elliptic systems under controllable growth condition, and obtain a general criterion for a weak solution to be regular in the neighborhood of a given boundary point. Combined with existing results on interior partial regularity, this result yields an upper bound on the Hausdorff dimension of the singular set at the boundary.

Keywords: quasilinear elliptic systems, controllable growth condition, A-harmonic approximation technique, boundary partial regularity

\section{Introduction}

In this article we are concerned with boundary regularity for weak solutions of quasilinear elliptic systems of the following type:

$$
-D_{\alpha}\left(A_{i j}^{\alpha \beta}(x, u) D_{\beta} u^{j}\right)=B^{i}(x, u, D u),
$$

where $\Omega$ is a bounded domain in $R^{n}, n \geq 2, u$ and $B^{i}$ take values in $R^{n}, N>1$. Here each $A_{i j}^{\alpha \beta}$ maps $\Omega \times R^{N}$ into $R$, and each $B^{i}$ maps $\Omega \times R^{N} \times R^{n N}$ into $R$. In this article, we shall be concerned with weak solutions. In order to define weak solutions to (1.1), one needs to impose certain structural and regularity conditions on $A_{i j}^{\alpha \beta}$ and inhomogeneity $B^{i}$ as follows:

(H1) There exists $L>0$ such that

$$
A_{i j}^{\alpha \beta}(x, \xi)(v, \tilde{v}) \leq L|v||\tilde{v}| \text { for all }(x, \xi) \in \bar{\Omega} \times R^{N}, v, \tilde{v} \in R^{n N} .
$$

(H2) $A_{i j}^{\alpha \beta}(x, \xi)$ is uniformly strongly elliptic, that is, for some $\lambda>0$ we have

$$
A_{i j}^{\alpha \beta}(x, \xi)(v, v) \geq \lambda|v|^{2} \quad \text { for all }(x, \xi) \in \bar{\Omega} \times R^{N}, v \in R^{n N} .
$$

(H3) There exists a monotone nondecreasing concave function $\omega(t, s):[0, \infty) \rightarrow[0$, $\infty)$ with $\omega(t, 0)=0$, continuous at 0 , such that

$$
\left|A_{i j}^{\alpha \beta}(x, u)-A_{i j}^{\alpha \beta}(y, v)\right| \leq \omega\left(|x-y|^{2}+|u-v|^{2}\right),
$$

for all $x, y \in \Omega, u, v \in R^{N}$. 
(H4) $B^{i}$ fulfill the following controllable growth condition:

$$
\left|B^{i}(x, \xi, v)\right| \leq C\left(|v|^{2\left(1-\frac{1}{r}\right)}+|\xi|^{r-1}+1\right),
$$

where $r=\frac{2 n}{n-2}$ if $n>2$, or any exponent if $n=2$; for all $x \in \bar{\Omega}, \xi \in R^{N}$ and $v \in R^{n N}$.

(H5) There exists $s$ with $s>n$ and a function $g \in H^{1, s}\left(\Omega, R^{n}\right)$, such that there holds:

$$
\left.u\right|_{\partial \Omega}=\left.g\right|_{\partial \Omega} .
$$

Note that we trivially have $g \in H^{1,2}\left(\Omega, R^{N}\right)$. Further, by Sobolev embedding theorem we have $g \in C^{0, \kappa}\left(\Omega, R^{n}\right)$ for any $\kappa \in\left[0,1-\frac{n}{s}\right]$. If $\left.g\right|_{\partial \Omega} \equiv 0$,we will take $g \equiv 0$ on $\Omega$.

We will be particularly concerned with a model case in which the domain is the upper half unit ball $B^{+}$. We reformulate the boundary condition for this case:

(H5)' There exists $s$ with $s>n$ and a function $g \in H^{1, s}\left(B^{+}, R^{n}\right)$ such that there holds:

$$
\left.u\right|_{D}=\left.g\right|_{D}
$$

For $x_{0} \in R^{n-1} \times\{0\}$ we write $D_{\rho}\left(x_{0}\right)=\left\{x \in R^{n}: x_{n}=0,\left|x-x_{0}\right|<\rho\right\}$, and set $D_{\rho}=$ $D_{\rho}(0), D=D_{1}$.

Definition 1.1. By a weak solution of (1.1) we mean a vector valued function $u \in W$ ${ }^{1,2}\left(\Omega, R^{n}\right)$ such that

$$
\int_{\Omega} A_{i j}^{\alpha \beta}(x, u)\left(D_{\beta} u^{j}, D_{\alpha} \varphi^{i}\right) d x=\int_{\Omega} B^{i}(x, u, D u) \cdot \varphi^{i} d x,
$$

holds for all test-functions $\varphi \in C_{0}^{\infty}\left(\Omega, R^{N}\right)$ and, by approximation, for all $\varphi \in W_{0}^{1,2}\left(\Omega, R^{N}\right)$. Where we have introduced the notation

$$
A_{i j}^{\alpha \beta}(x, \xi)(v, \tilde{v})=\left(A_{i j}^{\alpha \beta}(x, \xi) v\right) \cdot \tilde{v} .
$$

In the current situation the Sobolev embedding theorem yields the existence of a constant $C_{s}$ depending only on $s, n$ and $N$ such that there holds:

$$
\sup _{B_{\rho}^{+}\left(x_{0}\right)}\left|g-g_{x_{0}, \rho}^{\prime}\right| \leq C_{s} \rho^{1-\frac{n}{s}}\|g\|_{H^{1, s}\left(B_{\rho}^{+}\left(x_{0}\right), R^{N}\right)^{\prime}}
$$

for $x_{0} \in D, \rho \leq 1-\left|x_{0}\right|$. Obviously, the inequality remains true if we replace $\|g\|_{H^{1, s}\left(B_{\rho}^{+}\left(x_{0}\right), R^{N}\right)}$ by $\|g\|_{H^{1, s}\left(B^{+}, R^{N}\right)}$, which we will henceforth abbreviate simply as $\|g\|_{H^{1, s}}$

We also note here that the Poincare inequality in this setting yields:

$$
\int_{B_{\rho}^{+}\left(x_{0}\right)}\left|g-g_{x_{0}, \rho}^{\prime}\right|^{2} d x \leq C_{p} \rho^{2} \int_{B_{\rho}^{+}\left(x_{0}\right)}|D g|^{2} d x,
$$

for a constant $C_{p}$ which depending only on $n$.

Finally, we fix an exponent $\sigma \in(0,1)$ as follows: if $g \equiv 0, \sigma$ can be chosen arbitrary (but henceforth fixed); otherwise we take $\sigma$ fixed in $\left(0,1-\frac{n}{s}\right]$.

Under such assumptions on the structural conditions, full regularity of (1.1) at the boundary cannot, in general, be expected [1], even if the boundary data is smooth. Then, our goal is to establish a partial boundary regularity for weak solutions of systems (1.1). 
There are some previous partial regularity results for quasilinear systems. For example, Arkhipova has studied regularity up to the boundary for nonlinear and quasilinear systems [2-4]. And there are more results of particular forms. For systems in diagonal form, boundary regularity was first established by Wiegner [5], and the proof was generalized and extended by Hildebrandt-Widman [6]. The further discussions in this case can be seen in $[7,8]$. In the case of minima of functionals of the form $\int_{\Omega} A(x, u)|D u|^{2}$ $d x$, Jost-Meier [8] established full regularity in a neighborhood of the boundary. Further more, the dimension of singular set of solutions to non-differentiable elliptic systems are reduced by Kristensen and Mingione [9,10]

The result which is most closely related to that given here was shown that in [11] Grotowski obtained the boundary partial regularity results for more general systems of the form: $-D_{\alpha} A_{i}^{\alpha}(\cdot, u, D u)=f^{i}(\cdot, u, D u), i=1, \ldots, N$, under the quadratic growth and natural condition. And in [12], he got the analogous boundary regularity of (1.1) under natural growth condition. However the results in the current article do not follow from those in [11]; in the current situation we need only impose weaker structure conditions, and at the same time can obtain stronger conclusions.

The comparison is made possible by the technique of A-harmonic approximation. This technique is the natural extension of the technique of harmonic approximation. The harmonic approximation technique has its origins in Simon's [13] proof of the regularity theorem of Allard [14]. The technique of A-harmonic approximation then refers to the direct analog of the above situation. The interior version of this technique has previously been applied by Duzaar and Grotowski [15] to obtain a new, elementary proof for interior partial regularity for systems of the form (1.3). Then it has been extended and developed in [16,17].

In this article, we shown optimal boundary regularity of (1.1) under controllable growth condition. As the argument for combining the boundary and interior estimates is relatively standard, we omit it and get the following results:

Theorem 1.1. Let $\Omega$ be a bounded domain in $R^{n}$, with boundary of class $C^{1}$. Let $u$ be a weak solution of (1.1) satisfying the boundary condition (H5), where the structure conditions (H1)-(H3) hold for $A_{i j}^{\alpha \beta}$, and (H4) holds for $B^{i}$. Consider a fixed $\gamma \in(0, \sigma]$. Then there exist positive $R_{1}$ and $\varepsilon_{0}$ (depending only on $n, N, \lambda, L, \omega(\cdot)$ and $\gamma$ ) with the property that

$$
f_{B_{R}\left(x_{0}\right) \cap \Omega}\left|u-u_{x_{0}, R}^{\prime}\right|^{2} d x+\|g\|_{H^{1, s}}^{2} R^{2\left(1-\frac{n}{s}\right)}+R^{2} \leq \varepsilon_{0}^{2},
$$

for some $R \in\left(0, R_{1}\right]$ for a given $x_{0} \in \partial \Omega$ implies $u \in C^{0, \gamma}\left(\bar{B}_{\frac{R}{2}}\left(x_{0}\right) \cap \bar{\Omega}, R^{N}\right)$.

Note in particular that the boundary condition (H5) means that $u_{x_{0}, R}^{\prime}$ makes sense: in fact, we have $u_{x_{0}, R}^{\prime}=g_{x_{0}, R}^{\prime}$.

Combining this result with the analogous interior [18] and a standard covering argument allows us to obtain immediately the following bound on the size of the singular set:

Corollary 1.2. Under the assumptions of Theorem 1.1, the singular set of the weak solution $u$ has $(n-2)$-dimensional Hausdorff measure zero in $\bar{\Omega}$.

If the domain of the main step in proving Theorem 1.1 is a half ball; the result then follows from a relatively standard transformation argument. 
Theorem 1.2. Consider a weak solution of (1.1) on the upper half unit ball $B^{+}$which satisfies the boundary condition $(H 5)^{\prime}$, where the structure conditions $(\mathrm{H} 1)-(\mathrm{H} 3)$ hold for $A_{i j}^{\alpha \beta}$, and (H4) holds for $B^{i}$. Then there exist positive $R_{0}$ and $\varepsilon_{0}$ (depending only on $n$, $N, \lambda, L, \omega(\cdot)$ and $\gamma)$ with the property that

$$
f_{B_{R}^{+}\left(x_{0}\right)}\left|u-u_{x_{0}, R}^{\prime}\right|^{2} d x+\|g\|_{H^{1, s}}^{2} R^{2\left(1-\frac{n}{s}\right)}+R^{2} \leq \varepsilon_{0}^{2}
$$

for some $R \in\left(0, R_{0}\right]$ for a given $x_{0} \in D$ implies $u \in C^{0, \gamma}\left(\bar{B}_{\frac{R}{2}}\left(x_{0}\right), R^{N}\right)$.

Note that analogously to above, the boundary condition (H5)' ensures that $u_{x_{0}, R}^{\prime}$ exists, and we have indeed $u_{x_{0}, R}^{\prime}=g_{x_{0}, R}^{\prime}$.

We close this section by briefly summarizing the notation we use in this article. For a given set $X$ we denote by $L^{n}(X)$ and $H^{k}(X)$ its $n$-dimensional Lebesgue measure and $k$-dimensional Harsdorff measure, respectively. We write $B_{\rho}\left(x_{0}\right)=\left\{x \in R^{n}:\left|x-x_{0}\right|<\right.$ $\rho\}$, and further $B_{\rho}=B_{\rho}(0), B=B_{1}$. Similarly we denote upper half balls as follows: for $x_{0} \in R^{n-1} \times\{0\}$ we write $B_{\rho}^{+}\left(x_{0}\right)$ for $\left\{x \in R^{n}: x_{n}>0,\left|x-x_{0}\right|<\rho\right\}$, and set $B_{\rho}^{+}=B_{\rho}^{+}(0), B^{+}=B_{1}^{+}$. For bounded $X \subset R^{n}$ with $L^{n}(X)>0$ we denote the average of a given $g \in L^{1}$ by $f_{X} g d x$, i.e. $f_{X} g d x=\frac{1}{L^{n}(X)} \int_{X} g d x$. For $v \in L^{1}(\partial \Omega), x_{0} \in \partial \Omega$ we set $v_{x_{0}, R}^{\prime}=f_{\partial \Omega \cap \bar{B}_{R}\left(x_{0}\right)} v d H^{n-1}$. In particular, for $v \in L^{1}\left(D_{\rho}\left(x_{0}\right)\right), x_{0} \in D$, we write $v_{x_{0}, \rho}^{\prime}=f_{D_{\rho}\left(x_{0}\right)} v d H^{n-1}$. We let $\alpha_{n}$ denote the volume of the unit-ball in $R^{n}$, i.e. $\alpha_{n}=L^{n}$ $(B)$. We write $\operatorname{Bil}\left(R^{n N}\right)$ for the space of bilinear forms on the space $R^{n N}$.

\section{The A-harmonic approximation technique and some preliminary results}

In this section we present the A-harmonic approximation lemma [12], and some standard results due to Campanato $[19,20]$.

Lemma 2.1. (A-harmonic approximation lemma) Consider fixed positive $\lambda$ and $L$, and $n, N \in N$ with $n \geq 2$. Then for any given $\varepsilon>0$ there exists $\delta=\delta(n, N, \lambda, L, \varepsilon) \in$ $(0,1]$ with the following property: for any $A_{i j}^{\alpha \beta} \in \operatorname{Bil}\left(R^{n N}\right)$ satisfying

$$
A_{i j}^{\alpha \beta}(v, v) \geq \lambda|v|^{2}, \quad \text { for all } v \in R^{n N},
$$

and

$$
\mid A_{i j}^{\alpha \beta}\left(v, \bar{v}|\leq L| v|| \bar{v} \mid, \quad \text { for all } v, \bar{v} \in R^{n N},\right.
$$

for any $w \in H^{1,2}\left(B_{\rho}^{+}\left(x_{0}\right), R^{N}\right)$ (for some $\rho>0, x_{0} \in R^{n}$ ) satisfying

$$
\rho^{2-n} \int_{B_{\rho}^{+}\left(x_{0}\right)}|D w|^{2} d x \leq 1,
$$

and

$$
\left|\rho^{2-n} \int_{B_{\rho}^{+}\left(x_{0}\right)} A_{i j}^{\alpha \beta}(D g, D \varphi) d x\right| \leq \delta \rho \sup _{B_{\rho}^{+}\left(x_{0}\right)}|D \varphi|,
$$


and

$$
\left.w\right|_{D_{\rho}\left(x_{0}\right)}=0,
$$

for all $\varphi \in C_{0}^{1}\left(B_{\rho}^{+}\left(x_{0}\right), R^{N}\right)$,there exists an A-harmonic function

$$
v \in \tilde{H}=\left\{\left.\tilde{w} \in H^{1,2}\left(B_{\rho}^{+}\left(x_{0}\right), R^{N}\right)\left|\rho^{2-n} \int_{B_{\rho}^{+}\left(x_{0}\right)}\right| D \tilde{w}\right|^{2} d x \leq 1,\left.\tilde{w}\right|_{D_{\rho}\left(x_{0}\right)} \equiv 0\right\},
$$

with

$$
\rho^{-n} \int_{B_{\rho}^{+}\left(x_{0}\right)}|v-w|^{2} d x \leq \varepsilon .
$$

Next we recall a slight modification of a characterization of Hölder continuous functions [19].

Lemma 2.2. Consider $n \in N, n \geq 2$ and $x_{0} \in R^{n-1} \times\{0\}$. Suppose that there are positive constants $\kappa$ and $\alpha$, with $\alpha \in(0,1]$ such that, for some $v \in L^{2}\left(B_{6 R}^{+}\left(x_{0}\right)\right)$, there hold the following:

$$
\inf _{\mu \in R}\left\{\rho^{-n} \int_{B_{\rho}^{+}(\gamma)}|v-\mu|^{2} d x\right\} \leq \kappa^{2}\left(\frac{\rho}{R}\right)^{2 \alpha},
$$

for all $y \in D_{2 R}\left(x_{0}\right)$ and $\rho \leq 4 R$; and

$$
\inf _{\mu \in R} \rho^{-n}\left\{\int_{B_{\rho}(y)}|v-\mu|^{2} d x\right\} \leq \kappa^{2}\left(\frac{\rho}{R}\right)^{2 \alpha},
$$

for all $y \in B_{2 R}^{+}\left(x_{0}\right)$ and $B_{\rho}(y) \subset B_{2 R}^{+}\left(x_{0}\right)$.

Then there exists a Hölder continuous representatives of the $L^{2}$-class of $v$ on $\bar{B}_{R}^{+}\left(x_{0}\right)$, and for this representative $\bar{v}$ there holds:

$$
|\bar{v}(x)-\bar{v}(z)| \leq C_{\kappa}\left(\frac{|x-z|}{R}\right)^{\alpha},
$$

for all $x, z \in \bar{B}_{R}^{+}\left(x_{0}\right)$, for a constant $C_{\kappa}$ depending only on $n$ and $\alpha$.

We close this section by a standard estimate for the solutions to homogeneous second order elliptic systems with constant coefficients, due originally to Campanato [20].

Lemma 2.3. Consider fixed positive $\lambda$ and $L$, and $n, N \in N$ with $n \geq 2$. Then there exists $C_{0}$ depending only on $n, N, \lambda$ and $L$ (without loss of generality we take $C_{0} \geq 1$ ) such that for $A_{i j}^{\alpha \beta} \in \operatorname{Bil}\left(R^{n N}\right)$ satisfying (2.1) and (2.2), any $A$-harmonic function $h$ on $B_{\rho}^{+}\left(x_{0}\right)$ with $\left.h\right|_{D_{\rho}\left(x_{0}\right)} \equiv 0$ satisfies

$$
\rho^{2} \sup _{B_{\frac{\rho}{2}}^{+}\left(x_{0}\right)}|D h|^{2} \leq C_{0} \rho^{2-n} \int_{B_{\rho}^{+}\left(x_{0}\right)}|D h|^{2} d x .
$$




\section{Caccioppoli inequality}

In this section we prove the following Caccioppoli's inequality.

Theorem 3.1. Let $u \in W^{1,2}\left(\bar{\Omega}, R^{N}\right)$ be a weak solution of systems (1.1) under (H1)(H5). Then there exists $\rho_{0}>0, \rho_{0}$ depending only on $L, s$ and $\|g\|_{H^{1, s}}$, such that for all $B_{\rho}^{+}\left(x_{0}\right) \subset B^{+}$, with $x_{0} \in D^{+}, \rho<R<\rho_{0}$, there holds

$$
\begin{aligned}
& \int_{B_{\rho / 2}^{+}\left(x_{0}\right)}|D u|^{2} d x \leq \frac{C_{1}}{\rho^{2}} \int_{B_{\rho}^{+}\left(x_{0}\right)}\left|u(x)-u_{x_{0}, \rho}^{\prime}\right|^{2} d x+C_{2} \alpha_{n}\|g\|_{H^{1, s}}^{2} \rho^{n\left(1-\frac{2}{s}\right)} \\
& \quad+C_{3}\left(\int_{B_{\rho}^{+}\left(x_{0}\right)}\left(|D u|^{2}+|u|^{r}+1\right) d x\right)^{2\left(1-\frac{1}{r}\right)}
\end{aligned}
$$

where $C_{1}, C_{2}, C_{3}$ depending only on $\lambda, \mathrm{L}$ and $\|g\|_{L^{\infty}\left(B, R^{N}\right)^{\prime}}$ and $C_{2}$ additional on $C_{p}$, $C_{s}$, and also on $s$.

Proof. Now we consider a cut -off function $\eta \in C_{0}^{\infty}\left(B_{\rho / 2}^{+}\left(x_{0}\right)\right)$, satisfying $0 \leq \eta \leq 1, \eta \equiv 1$ on $B_{\rho / 2}^{+}\left(x_{0}\right)$ and $|\nabla \eta|<\frac{4}{\rho}$. Then the function $(u-g) \eta^{2}$ is in $W_{0}^{1,2}\left(B_{\rho / 2}^{+}\left(x_{0}, R^{N}\right)\right)$, and thus can be taken as a test-function.

Using (1.2), (H1), (H4), (H5), for $\varepsilon$ positive but arbitrary (to be fixed later), we have:

$$
\begin{aligned}
& \int_{B_{\rho}^{+}\left(x_{0}\right)} A_{i j}^{\alpha \beta}(x, u)(D u, D u) \eta^{2} d x \\
& =-2 \int_{B_{\rho}^{+}\left(x_{0}\right)} A_{i j}^{\alpha \beta}(x, u)(D u, D \eta)(u-g) \eta d x \\
& +\int_{B_{\rho}^{+}\left(x_{0}\right)} A_{i j}^{\alpha \beta}(x, u)(D u, D g) \eta^{2} d x+\int_{B_{\rho}^{+}\left(x_{0}\right)} B^{i}(x, u, D u) \cdot \varphi^{i} d x \\
& \leq 2 L \int_{B_{\rho}^{+}\left(x_{0}\right)}|D u||D \eta \| u-g| \eta d x+L \int_{B_{\rho}^{+}\left(x_{0}\right)}|D u||D g| \eta^{2} d x \\
& +C \int_{B_{\rho}^{+}\left(x_{0}\right)}\left(|D u|^{2\left(1-\frac{1}{r}\right)}+|u|^{r-1}+1\right) \cdot \varphi d x \\
& \leq 2 \varepsilon \int_{B_{\rho}^{+}\left(x_{0}\right)}|D u|^{2} \eta^{2} d x+\frac{L^{2}}{\varepsilon} \int_{B_{\rho}^{+}\left(x_{0}\right)}|u-g|^{2}|D \eta|^{2} d x+\frac{L^{2}}{\varepsilon} \int_{B_{\rho}^{+}\left(x_{0}\right)}|D g|^{2} d x \\
& +C\left(\int_{B_{p}^{+}\left(x_{0}\right)}\left(|D u|^{2}+|u|^{r}+1\right) d x\right)^{\left(1-\frac{1}{r}\right)}\left(\int_{B_{p}^{+}\left(x_{0}\right)}|\varphi|^{r} d x\right)^{\frac{1}{r}} \\
& \leq 2 \varepsilon \int_{B_{\rho}^{+}\left(x_{0}\right)}|D u|^{2} \eta^{2} d x+\frac{L^{2}}{\varepsilon} \int_{B_{\rho}^{+}\left(x_{0}\right)}|u-g|^{2}|D \eta|^{2} d x+\frac{L^{2}}{\varepsilon} \int_{B_{\rho}^{+}\left(x_{0}\right)}|D g|^{2} d x \\
& +C\left(\int_{B_{\rho}^{+}\left(x_{0}\right)}\left(|D u|^{2}+|u|^{r}+1\right) d x\right)^{\left(1-\frac{1}{r}\right)}\left(\int_{B_{\rho}^{+}\left(x_{0}\right)}|D \varphi|^{2} d x\right)^{\frac{1}{2}} \\
& \leq 3 \varepsilon \int_{B_{\rho}^{+}\left(x_{0}\right)}|D u|^{2} \eta^{2} d x+\left(\frac{L^{2}}{\varepsilon}+C(\varepsilon)\right) \int_{B_{\rho}^{+}\left(x_{0}\right)}|u-g|^{2}|D \eta|^{2} d x+C(\varepsilon) \int_{B_{\rho}^{+}\left(x_{0}\right)}|D g|^{2} d x \\
& +C(\varepsilon)\left(\int_{B_{\rho}^{+}\left(x_{0}\right)}\left(|D u|^{2}+|u|^{r}+1\right) d x\right)^{2\left(1-\frac{1}{r}\right)} .
\end{aligned}
$$


Using (H2) and the fact that $u_{x_{0}, \rho}^{\prime}=g_{x_{0}, \rho}^{\prime}$, we thus have:

$$
\begin{aligned}
& \lambda \int_{B_{\rho}^{+}\left(x_{0}\right)}|D u|^{2} \eta^{2} d x \\
& \leq\left. 3 \varepsilon \int_{B_{\rho}^{+}\left(x_{0}\right)} D u\right|^{2} \eta^{2} d x+2\left(\frac{L^{2}}{\varepsilon}+C(\varepsilon)\right) \int_{B_{\rho}^{+}\left(x_{0}\right)}\left|u-u_{x_{0}, \rho}^{\prime}\right|^{2}|D \eta|^{2} d x+C(\varepsilon) \int_{B_{\rho}^{+}\left(x_{0}\right)}|D g|^{2} d x \\
& \quad+2\left(\frac{L^{2}}{\varepsilon}+C(\varepsilon)\right) \int_{B_{\rho}^{+}\left(x_{0}\right)}\left|g-g_{x_{0}, \rho}^{\prime}\right|^{2}|D \eta|^{2} d x+C(\varepsilon)\left(\int_{B_{\rho}^{+}\left(x_{0}\right)}\left(|D u|^{2}+|u|^{r}+1\right) d x\right)^{2\left(1-\frac{1}{r}\right)} .
\end{aligned}
$$

Thus, by (1.4), (1.5), we can get:

$$
\begin{aligned}
& (\lambda-3 \varepsilon) \int_{B_{\rho}^{+}\left(x_{0}\right)}|D u|^{2} \eta^{2} d x \leq 2\left(\frac{L^{2}}{\varepsilon}+C(\varepsilon)\right) \int_{B_{\rho}^{+}\left(x_{0}\right)}\left|u-u_{x_{0}, \rho}^{\prime}\right|^{2}|D \eta|^{2} d x \\
& +\left[2\left(\frac{L^{2}}{\varepsilon}+\varepsilon\right) C_{p}+C(\varepsilon)\right] \alpha_{n}\|g\|_{H^{1, s}}^{2} \rho^{n\left(1-\frac{2}{s}\right)}+C(\varepsilon)\left(\int_{B_{\rho}^{+}\left(x_{0}\right)}\left(|D u|^{2}+|u|^{r}+1\right) d x\right)^{2\left(1-\frac{1}{r}\right)} .
\end{aligned}
$$

Fix $\varepsilon$ small enough such that $\lambda-3 \varepsilon>0$, thus (3.3) yields the desired inequality, where $C_{1}, C_{2}, C_{3}$ depending only on $\|g\|_{L^{\infty}\left(B, R^{N}\right)^{\prime}} \lambda$ and $L$, and $C_{2}$ additional on $C_{p}, C_{s}$ and also on $s$.

\section{The proof of the main theorem}

In this section we proceed to the proof of partial regularity result, and hence consider $u \in W^{1,2}\left(\Omega, R^{n}\right)$ to be a weak solution of (1.1). For $R<1-\left|x_{0}\right|, x_{0} \in D, y \in D_{R}\left(x_{0}\right)$, $D_{\rho}(y) \subset \subset D_{R}\left(x_{0}\right)$, and $\varphi \in C_{0}^{\infty}\left(B_{\frac{\rho}{2}}^{+}(y), R^{N}\right)$ with $\sup _{B_{\rho}^{+}(\gamma)}|D \varphi| \leq 1$, we have

\section{Lemma 4.1.}

$$
\left|\left(\frac{\rho}{2}\right)^{2-n} \int_{B_{\frac{\rho}{2}}^{+}(y)} A\left(y, u_{\gamma, \rho}^{+}\right)(D u, D \varphi) d x\right| \leq C_{8} \frac{\rho}{2} \sqrt{I}\left(\sqrt{I}+\omega^{\frac{1}{2}}(I)\right) \sup _{B_{\frac{\rho}{2}}^{+}\left(x_{0}\right)}|D \varphi|,
$$

here and hereafter, we define

$$
I\left(z, r_{0}\right)=f_{B_{r_{0}}^{+}(z)}\left|u-u_{z, r_{0}}^{\prime}\right|^{2} d x+\|g\|_{H^{1, s}}^{2} r_{0}^{2(1-n / s)}+r_{0}^{2},
$$

for $z \in D, r_{0} \in(0,1-|z|)$.

Proof. Consider $x_{0} \in D$ and $y \in D_{R}\left(x_{0}\right), D_{\rho}(y) \subset \subset D_{R}\left(x_{0}\right)$, for $R<1-\left|x_{0}\right|$, and $\varphi \in C_{0}^{\infty}\left(B_{\frac{\rho}{2}}^{+}(y), R^{N}\right)$ with $\sup _{B_{\rho}^{+}(y)}|D \varphi| \leq 1$. From the definition of weak solution:

$$
\begin{aligned}
& \int_{B_{\frac{\rho}{2}}^{+}(\gamma)} A_{i j}^{\alpha \beta}\left(y, u_{\gamma, \rho}^{\prime}\right)(D u, D \varphi) d x \\
& \leq C\left[\int_{B_{\frac{\rho}{2}}^{+}(y)}\left(|D u|^{2\left(1-\frac{1}{r}\right)}+|u|^{r-1}+1\right) d x\right] \rho \sup _{B_{\frac{\rho}{2}}^{+}(\gamma)}|D \varphi| \\
& +\int_{B_{\frac{\rho}{2}}^{+}(y)}\left|A_{i j}^{\alpha \beta}\left(y, u_{\gamma, \rho}^{\prime}\right)-A_{i j}^{\alpha \beta}(x, u)\right||D u| d x \sup _{B_{\frac{\rho}{2}}^{+}(y)}|D \varphi| .
\end{aligned}
$$


By Sobolev's and Hölder's inequalities, and then Young's inequality, we have

$$
\begin{aligned}
& C \int_{B_{\frac{\rho}{2}}^{+}(y)}\left(|D u|^{2\left(1-\frac{1}{r}\right)}+|u|^{r-1}+1\right) d x \\
& \leq C\left(\int_{B_{\frac{\rho}{2}}^{+}(y)}|D u|^{2} d x\right)^{1-\frac{1}{r}}\left(\int_{B_{\frac{\rho}{2}}^{+}(y)} d x\right)^{\frac{1}{r}}+C\left(\left|u_{\gamma, \rho / 2}^{\prime}\right|^{r-1}+1\right) \alpha_{n} \rho^{n} / 2 \\
& +C\left(\int_{B_{\frac{\rho}{2}}^{+}(\gamma)}\left|u-u_{\gamma, \rho / 2}^{\prime}\right|^{r} d x\right)^{\left(1-\frac{1}{r}\right)}\left(\int_{B_{\frac{\rho}{2}}^{+}(\gamma)} d x\right)^{\frac{1}{r}} \\
& \leq C\left(\int_{B_{\frac{\rho}{2}}^{+}(y)}|D u|^{2} d x\right)^{1-\frac{1}{r}}\left(\int_{B_{\frac{\rho}{2}}^{+}(y)} d x\right)^{\frac{1}{r}}+C\left(\left|u_{\gamma, \rho / 2}^{\prime}\right|^{r-1}+1\right) \alpha_{n} \rho^{n} / 2 \\
& +C\left(\int_{B_{\frac{\rho}{2}}^{+}(\gamma)}|D u|^{2} d x\right)^{\frac{r}{2}\left(1-\frac{1}{r}\right)}\left(\int_{B_{\frac{\rho}{2}}^{+}(\gamma)} d x\right)^{\frac{1}{r}} \\
& \leq C\left(\int_{B_{\frac{\rho}{2}}^{+}(\gamma)}|D u|^{2} d x\right)^{1-\frac{1}{r}}\left(\int_{B_{\frac{\rho}{2}}^{+}(\gamma)} d x\right)^{\frac{1}{r}}+C\left(\left|u_{\gamma, \rho / 2}^{\prime}\right|^{r-1}+1\right) \alpha_{n} \rho^{n} / 2 \\
& +C\left(\int_{B_{\frac{\rho}{2}}^{+}(y)}|D u|^{2} d x\right)^{\left(\frac{r}{2}-1\right)\left(1-\frac{1}{r}\right)}\left(\int_{B_{\frac{\rho}{2}}^{+}(y)}|D u|^{2} d x\right)^{1-\frac{1}{r}}\left(\int_{B_{\frac{\rho}{2}}^{+}(y)} d x\right)^{\frac{1}{r}} \\
& \leq C\left(\|u\|_{W^{1,2}}\right)\left(\int_{B_{\frac{\rho}{2}}^{+}(y)}|D u|^{2} d x\right)^{1-\frac{1}{r}}\left(\int_{B_{\frac{\rho}{2}}^{+}(\gamma)} d x\right)^{\frac{1}{r}}+C\left(\left|u_{y, \rho / 2}^{\prime}\right|^{r-1}+1\right) \frac{\alpha_{n} \rho^{n}}{2} \\
& \leq \varepsilon \int_{B_{\frac{\rho}{2}}^{+}(y)}|D u|^{2} d x+C\left(\varepsilon,\|u\|_{W^{1,2}}\right)\left(\left|u_{\gamma, \rho / 2}^{\prime}\right|^{r-1}+1\right) \alpha_{n} \rho^{n} / 2
\end{aligned}
$$

and

$$
\begin{aligned}
& C_{3}\left(\int_{B_{\rho}^{+}\left(x_{0}\right)}\left(|D u|^{2}+|u|^{r}+1\right) d x\right)^{2\left(1-\frac{1}{r}\right)} \\
& \leq \frac{c_{4}}{2} \alpha_{n} \rho^{n+2}\left(f_{B_{\rho}^{+}\left(x_{0}\right)}\left(|D u|^{2}+\left|u-u_{x_{0}, \rho}^{\prime}\right|^{r}+\left|u_{x_{0}, \rho}^{\prime}\right|+1\right) d x\right)^{2\left(1-\frac{1}{r}\right)} \\
& \leq C_{5} \alpha_{n} \rho^{n+2}\left[\left(f_{B_{\rho}^{+}\left(x_{0}\right)}|D u|^{2} d x\right)^{2\left(1-\frac{1}{r}\right)}+\left(f_{B_{\rho}^{+}\left(x_{0}\right)}|D u|^{2} d x\right)^{r\left(1-\frac{1}{r}\right)}+\left(\left|u_{x_{0}, \rho}^{\prime}\right|+1\right)^{2\left(1-\frac{1}{r}\right)}\right] \\
& \leq C_{6}\left(\left.|| u\right|_{W^{1,2},}\left|u_{x_{0}, \rho}^{\prime}\right|\right) \alpha_{n} \rho^{n+2},
\end{aligned}
$$

where we have used $r=\frac{2 n}{n-2}$ and $u \in W^{1,2}\left(\bar{\Omega}, R^{N}\right)$. 
Then Caccioppoli inequality yields

$$
\int_{B_{\rho / 2}^{+}\left(x_{0}\right)}|D u|^{2} d x \leq \frac{C_{1}}{\rho^{2}} \int_{B_{\rho}^{+}\left(x_{0}\right)}\left|u(x)-u_{x_{0}, \rho}^{\prime}\right|^{2} d x+C_{2} \alpha_{n}\|g\|_{H^{1, s}}^{2} \rho^{n\left(1-\frac{2}{s}\right)}+C_{6} \alpha_{n} \rho^{n+2}(4.4)
$$

Henceforth we restrict to $\rho$ sufficiently small. Applying in turn Young's inequality, (H3), (4.4) and Jensen's inequality we calculate from (4.2):

$$
\begin{aligned}
& \int_{B_{\frac{\rho}{2}}^{+}(\gamma)} A_{i j}^{\alpha \beta}\left(y, u_{\gamma, \rho}^{\prime}\right)(D u, D \varphi) d x \\
& \leq C \rho\left[\int_{B_{\frac{\rho}{2}}^{+}(y)}|D u|^{2} d x+\left(\left|u_{y, \rho / 2}^{\prime}\right|^{r-1}+1\right) \alpha_{n} \rho^{n} / 2\right] \\
& +\left[\int_{B_{\frac{\rho}{2}}^{+}(\gamma)}\left|A_{i j}^{\alpha \beta}\left(y, u_{\gamma, \rho}^{\prime}\right)-A_{i j}^{\alpha \beta}(x, u)\right|^{2} d x\right]^{\frac{1}{2}} \cdot\left[\int_{B_{\frac{\rho}{2}}^{+}(y)}|D u|^{2} d x\right]^{\frac{1}{2}} \\
& \leq C \rho\left[\int_{B_{\frac{\rho}{2}}^{+}(\gamma)}|D u|^{2} d x+\left(\left|u_{y, \rho / 2}^{\prime}\right|^{r-1}+1\right) \alpha_{n} \rho^{n} / 2\right] \\
& +\omega^{\frac{1}{2}}\left(\rho^{2}+f_{B_{\rho}^{+}(y)}\left|u-u_{\gamma, \rho}^{\prime}\right|^{2} d x\right)\left(\alpha_{n} \rho^{n} / 2\right)\left(f_{B_{\rho}^{+}(y)}|D u|^{2} d x\right)^{\frac{1}{2}} \text {. }
\end{aligned}
$$

For $z \in D, \quad r_{0} \in(0,1-|z|)$. We introduce the $\operatorname{notation} I\left(z, r_{0}\right)=f_{B_{r_{0}}^{+}(z)}\left|u-u_{z, r_{0}}^{\prime}\right|^{2} d x+\|g\|_{H^{1, s}}^{2} r_{0}^{2(1-n / s)}+r_{0}^{2}$,

and further write $I$ for $I(y, \rho)$. We have from (4.5) and by Poincare's inequality:

$$
\int_{B_{\frac{\rho}{2}}^{+}(\gamma)} A_{i j}^{\alpha \beta}\left(y, u_{\gamma, \rho}^{\prime}\right)(D u, D \varphi) d x \leq \frac{C_{7}}{2} \alpha_{n} \rho^{n-1}\left[I+\omega^{\frac{1}{2}}(I) I^{\frac{1}{2}}\right] .
$$

For arbitrary $\varphi \in C_{0}^{\infty}\left(\Omega, R^{N}\right)$ we thus have, by recalling:

$$
\int_{B_{\frac{\rho}{2}}^{+}(\gamma)} A_{i j}^{\alpha \beta}\left(y, u_{\gamma, \rho}^{\prime}\right)(D u, D \varphi) d x \leq \frac{C_{7}}{2} \alpha_{n} \rho^{n-1}\left[I+\omega^{\frac{1}{2}}(I) I^{\frac{1}{2}}\right] \sup _{B_{\frac{\rho}{2}}^{+}\left(x_{0}\right)}|D \varphi| .
$$

Multiplying through by $\left(\frac{\rho}{2}\right)^{2-n}$, this yields:

$$
\left|\left(\frac{\rho}{2}\right)^{2-n} \int_{B_{\frac{\rho}{2}}^{+}(y)} A_{i j}^{\alpha \beta}\left(y, u_{\gamma, \rho}^{\prime}\right)(D u, D \varphi) d x\right| \leq C_{8} \frac{\rho}{2}\left[I+\omega \frac{1}{2}(I) I \frac{1}{2}\right] \sup _{B_{\frac{\rho}{2}}^{+}\left(x_{0}\right)}|D \varphi|,
$$

for $C_{8}$ define by $C_{8}=2^{n-3} C_{7} \alpha_{n}$.

Lemma 4.2. Consider $u$ satisfying the condition of Theorem 1 and $\sigma$ fixed, then we can find $\delta$ and $s_{0}$ together with positive constants $C_{11}$ such that the smallness conditions: 


$$
0<\omega\left(|u|, s_{0}\right) \leq\left(\frac{\delta}{2}\right)^{2}
$$

and

$$
I\left(x_{0}, R\right) \leq C_{13}^{-1} \min \left\{\frac{\delta^{2}}{4}, s_{0}\right\},
$$

together imply the growth condition

$$
I(\gamma, \theta \rho) \leq \theta^{2 \sigma} I(\gamma, \rho) .
$$

Proof. Combining Lemma 2.1 and Theorem 3.1, and applying Lemma 2.2, we can conclude the desired Hölder continuity. As the argument is relatively standard and similarly to [12], we omit it.

\section{Acknowledgements}

This article was supported by the National Natural Science Foundation of China (Nos: 10976026; 61101239); the Natural Science Foundation of Zhejiang Province (Y6110078) and Program for New Century Excellent Talents in Zhangzhou Normal University (No: SX1102)

\section{Author details}

${ }^{1}$ Department of Mathematics and Information Science, Zhangzhou Normal University, Zhangzhou 363000, Fujian, China ${ }^{2}$ School of Mathematical Science, Xiamen University, Xiamen 361005, Fujian, China

\section{Authors' contributions}

Shuhong Chen participated in design of the study and drafted the manuscript. Zhong Tan participated in conceived of the study and the amendment of the paper. All authors read and approved the final manuscript.

\section{Competing interests}

The authors declare that they have no competing interests.

Received: 29 October 2011 Accepted: 30 April 2012 Published: 30 April 2012

\section{References}

1. Giaquinta, M: A counter-example to the boundary regularity of solutions to elliptic quasilinear systems. Manus Math. 24 217-220 (1978). doi:10.1007/BF01310055

2. Arkhipova, AA: Regularity results for quasilinear elliptic systems with nonlinear boundary conditions. J Math Sci. 77(4):3277-3294 (1995). doi:10.1007/BF02364861

3. Arkhipova, AA: On the regularity of solutions of boundary-value problem for quasilinear elliptic systems with quadratic nonlinear. J Math Sci. 80(6):2208-2225 (1995)

4. Arkhipova, AA: On the regularity of the oblique derivative problem for quasilinear elliptic systems. J Math Sci. 84(1):817-822 (1997). doi:10.1007/BF02399934

5. Wiegner, M: A priori schranken für Lösungen gewisser elliptischer systeme. Manuscr Math. 18, 279-297 (1976) doi:10.1007/BF01245922

6. Hildebrandt, S, Widman, KO: On the hölder continuity of weak solutions of quasilinear elliptic systems of second order. Ann Sci Norm Sup Pisa IV. 4, 144-178 (1977)

7. Hildebrandt, S: Nonlinear elliptic systems and harmonic mappings. Proceedings, 1980 Beijing Symposium on Differential Geometry and Differential Equations.1, 481-615

8. Jost, J, Meier, M: Boundary regularity for minima of certain quadratic functionals. Math Ann. 262, 549-561 (1983). doi:10.1007/BF01456068

9. Kristensen, J, Mingione, G: The singular set of Lipschitizan minima of multiple integrals. Arch Rat Mech Anal. 184 341-369 (2007). doi:10.1007/s00205-006-0036-2

10. Mingione, G: The singular set of solutions to non-differentiable elliptic systems. Arch Rat Mech Anal. 166, 287-301 (2003). doi:10.1007/s00205-002-0231-8

11. Grotowski, JF: Boundary regularity for nonlinear elliptic systems. Calc Var Partial Di Diff Equ. 15(3):353-388 (2002). doi:10.1007/s005260100131

12. Grotowski, JF: Boundary regularity for quasilinear elliptic systems. Commun Partial Diff Equ. 27(11-12):2491-2512 (2002). doi:10.1081/PDE-120016165

13. Simon, L: Lectures on Geometric Measure Theory. Australian National University Press, Canberra (1983)

14. Allard, WK: On the first variation of a varifold. Ann Math. 95, 225-254 (1972)

15. Duzaar, F, Grotowski, JF: Partial regularity for nonlinear elliptic systems: the method of A-harmonic approximation. Manuscr Math. 103, 267-298 (2000). doi:10.1007/s002290070007

16. Duzaar, F, Steffen, K: Optimal interior and boundary regularity for almost minimal currents to elliptic integrands. J Reine Angew Math. 546, 73-138 (2002) 
17. Grotowski, JF: Boundary Regularity Results for Nonlinear Elliptic Systems in Divergence Form. (2000) Habilitationsschrift, Friedrich-Alexander-Universit?ä?t Erlangen-N?ü?rnberg

18. Giaquinta, M: Multiple Integrals in the Calculus of Variations and Nonlinear Elliptic Systems. Princeton University Press, Princeton, NJ (1983)

19. Campanato, S: Proprietà di Hölderianità di alcune classi di funzioni. Ann Sci Norm Sup Pisa Ser III. 17, 175-188 (1963)

20. Campanato, S: Equazioni ellittiche del Ifordine e spazi $L^{2 \lambda}$. Ann Mat Pura Appl. 69, $321-381$ (1965). doi:10.1007/ BF02414377

doi:10.1186/1029-242X-2012-102

Cite this article as: Chen and Tan: Boundary regularity result for quasilinear elliptic systems. Journal of Inequalities and Applications 2012 2012:102.

\section{Submit your manuscript to a SpringerOpen ${ }^{\odot}$} journal and benefit from:

- Convenient online submission

- Rigorous peer review

- Immediate publication on acceptance

- Open access: articles freely available online

- High visibility within the field

- Retaining the copyright to your article

Submit your next manuscript at $\boldsymbol{s p r i n g e r o p e n . c o m ~}$ 